\title{
A EQUAÇÃO DO CALOR UNIDIMENSIONAL E BIDIMENSIONAL E APLICAÇÕES
}

\author{
Pedro Álvares Muiños*, Bianca Morelli Rodolfo Calsavara
}

\section{Resumo}

Neste projeto foram estudadas existência de solução da equação do calor nos casos unidimensional e bidimensional, assim como algumas propriedades e aplicações. As propriedades estudadas para as soluções da equação do calor foram principios de máximo, unicidade, dependência contínua da solução em relação as condições iniciais e regularidade. Em particular, no caso unidimensional foram estudadas aplicações como problemas de condução de calor em barras infinitas e finitas.

Palavras-chave:

Equações diferenciais parciais, equação do calor, equações parabólicas.

\section{Introdução}

As equações diferenciais possuem uma ampla gama de aplicações que abrangem desde a física e engenharia até a biologia e administração. Isto se deve ao fato de que os fenômenos estudados por essas e outras áreas podem ser modelados utilizando tais equações.

Neste projeto, foram estudadas existência, unicidade e algumas propriedades da equação do calor unidimensional. A partir daí, foi realizado um estudo sobre aplicações da equação do calor, sendo analisados problemas de condução de calor em barras finitas e infinitas com distintas condições iniciais e de contorno.

Além disso, foram estudadas existência, unicidade e algumas propriedades da equação do calor bidimensional. Sendo que estes estudos foram generalizados para o caso $n$-dimensional $(n \geq 2)$.

\section{Resultados e Discussão}

Inicialmente, foi realizado um estudo preliminar sobre série de funções, convergência e convergência uniforme. Com isso, puderam ser estudadas convergência e propriedades de séries de Fourier.

Em seguida, foi realizada a dedução da equação do calor unidimensional. Então, foram estudados problemas de condução de calor em barras infinitas e finitas. Utilizando o método de separação de variáveis foram encontradas soluções do problema de condução de calor homogêneo em uma barra finita. Neste caso, foram estudadas diversas condições de contorno como as do tipo Dirichlet e Neumann. Posteriormente, foram estudados problemas de valor inicial e de contorno para alguns casos de equação não homogênea.

Após provar a existência de solução para problemas de condução de calor homogêneo foi demonstrada a unicidade da solução para tais problemas. Para isso, foi utilizado o princípio de máximo para a equação do calor unidimensional. Além disso, foi estudado a dependência contínua da solução em relação as condições iniciais.

Posteriormente, foi provada a existência da solução para problemas de valor inicial e de contorno envolvendo a equação do calor bidimensional homogênea. Como no caso da equação do calor unidimensional, foi demonstrada a unicidade da solução para problemas de valor inicial usando o princípio de máximo para a equação do calor bidimensional. O princípio de máximo, por sua vez, foi provado utilizando a fórmula do valor médio para equação do calor. Em seguida, foi provada a regularidade da solução da equação do calor e estudadas algumas estimativas de suas derivadas. $\mathrm{E}$, por último, foi demonstrada novamente a unicidade da solução para o caso bidimensional, desta vez usando o método da energia.

\section{Conclusões}

Para a equação do calor unidimensional e $n$ dimensional ( $n \geq 2$ ) foram obtidos resultados sobre existência e unicidade de solução, além de propriedades de solução desta equação. Também foram estudadas aplicações da equação do calor unidimensional, como problemas de condução de calor em barras finitas e infinitas com distintas condições iniciais e de contorno.

Durante a execução do projeto foram aprofundados conhecimentos já estudados em disciplinas cursadas durante a graduação pelo aluno. Além disso, o aluno foi introduzido a novos conceitos e ao estudo de aplicações de equações diferenciais parciais.

Com isso, o aluno desenvolveu a capacidade de trabalhar com resultados teóricos na área de equações diferenciais parciais e habilidades com relação a manipulação de tais equações. Adquirindo assim conhecimento para prosseguir com estudos mais avançados em assuntos que envolvam equações diferenciais parciais.

\section{Agradecimentos}

À minha orientadora, Bianca Morelli Rodolfo Calsavara, pelo apoio durante todo o projeto.

Ao CNPq pela bolsa concedida para realização do trabalho.

${ }^{1}$ L. Evans, Partial Differential Equations, American Mathematical Society, 1998.

2 D. J. Figueiredo, Análise de Fourier e Equações Diferenciais Parciais, 4a edição, Projeto Euclides, IMPA, Rio de Janeiro, 2005.

${ }^{3}$ H. L. Guidorizzi, Cálculo, Vol. 4, 5a edição, Ed. LTC, 2004.

4 S. J. Farlow, Partial Differential Equations for Scientists and Engineers, Editora Dover, 1993.

5 V. Iório, EDP Um Curso de Graduação, 2a edição, Coleção Matemática Universitária, IMPA, Rio de Janeiro, 2005. 